\title{
La resolución de problemas con números enteros. Una experiencia con estudiantes de séptimo grado de Educación Media
}

\section{Solving problems with integers numbers. An experience with seventh grade student in middle education}

\author{
LANCHEROS, Jhon ${ }^{1}$ \\ GUALDRÓN, Elgar ${ }^{2}$ \\ ÁVILA, Adriana ${ }^{3}$
}

\begin{abstract}
Resumen
El presente artículo describe una investigación fundamentada en el diseño e implementación de una secuencia didáctica basada en el método de Pólya y la aplicación de la metodología del Aprendizaje Basado en Problemas (ABP) como estrategia para fortalecer la competencia de resolución de problemas en el grado séptimo de la educación media. Los resultados sugieren que la estrategia usada fue útil al docente para la identificación de dificultades, ritmos de aprendizaje y fortalezas de los estudiantes.

Palabras clave: secuencia didáctica, aprendizaje basado en problemas, método de Pólya, competencia resolución de problemas.
\end{abstract}

\begin{abstract}
This papper described a research that is based on the implementation and design of a didactic sequence based on the Pólya's method and the application of the Problem Based Learning (ABP) methodology as a strategy to strengthen problem-solving competence in the seventh grade in middle education. The results suggest that the strategy used was useful to the teacher for the identification of difficulties, learning rhythms and strengths of the students.

Key words: didactic sequence, problem based learning, Pólya's method, problem solving competence.
\end{abstract}

\section{Introducción}

La resolución de problemas es una competencia fundamental en el área de matemáticas que implica enfrentar múltiples situaciones, aprender y aplicar sus conocimientos para buscar e implementar posibles soluciones (Cockcroft, 1982; OECD, 2006; MEN, 1998), lo cual requiere de flexibilidad y apertura a alternativas no necesariamente conocidas, implicando un nivel alto de complejidad para los estudiantes (Pólya, 1989; Callejo, 1998), incluso para algunos docentes esta es vista como un tema de estudio y no como una capacidad que debe

\footnotetext{
${ }^{1}$ Estudiante Maestría en Educación. Facultad de Ciencias Sociales, Humanidades y Artes. Universidad Autónoma de Bucaramanga. jlancheros743@unab.edu.co

2 Doctor en Didáctica de las Matemáticas. Docente de la Universidad de Pamplona (Colombia), Departamento de Matemáticas. Director del grupo de Investigación EDUMATEST_Universidad de Pamplona. egualdron@unipamplona.edu.co

${ }^{3}$ Doctora en Educación. Docente de la Facultad de Ciencias Sociales, Humanidades y Artes. Universidad Autónoma de Bucaramanga. aavila2@unab.edu.co
} 
desarrollarse en el individuo, por lo que se considera un contenido adicional a la enseñanza de las matemáticas, y que debe enseñarse por separado. Otro de los errores comunes en las instituciones educativas, es que el docente no emplea estrategias para su aplicación práctica, por lo que concluyen que las situaciones problemáticas no se pueden asociar al contexto educativo. Lo anterior es contrario a lo expuesto por el Ministerio de Educación Nacional de Colombia, MEN (1998), que resalta el contexto en las diferentes fases del aprendizaje, ya que permite al estudiante descubrir o reinventar las matemáticas. En este sentido, las estrategias que se pretendan aplicar deben estar orientadas a facilitar a los estudiantes las herramientas y habilidades para hacer dicha transferencia; lo anterior se debe, en parte, a la falta de planeación por parte de los maestros, pues la aplicación se deja para el final del programa formativo, razón por la cual se suelen omitir por falta de tiempo.

Es esencial que, tanto docentes como estudiantes, desarrollen y fortalezcan sus capacidades en la creación y resolución de problemas, teniendo en cuenta que estos deben cumplir ciertas características: que correspondan a la realidad (Valdez-Rojo \& Tobón, 2018), y que favorezcan la aparición de conceptos y permitan construir sentido a lo estudiado. Resulta claro que una buena selección de situaciones problemáticas contribuye de gran manera en el aprendizaje de los estudiantes, debido a que despiertan sus intereses, los motiva a investigar y proponer nuevas estrategias de solución, los invita a ir más allá del problema y a plantearse nuevos retos (actividad que muy pocos estudiantes realizan).

El ABP es un método de enseñanza aprendizaje inspirado en la estrategia de enseñanza llamada aprendizaje por descubrimiento y construcción, que tiene como principal característica el apartarse de la estrategia expositiva por parte del profesor y se centra en el desarrollo de conocimientos, habilidades y actitudes de los estudiantes a través de situaciones de la vida real (Restrepo, 2005), que involucra de manera plena la participación activa de los estudiantes (Alvarez, 2020).

El método heurístico de George Pólya, plasmado desde tres de sus principales publicaciones: "Cómo resolverlo" (1945/1985), "Descubrimiento matemático" (1962/1981) y “Matemáticas y razonamiento plausible" (1954a, 1954b), se articula desde la premisa que el aprendizaje debe ser activo y atractivo, donde el trabajo del profesor de matemáticas ayude a los estudiantes a "descubrir por sí mismos tanto como sea posible" (1962/1981, p. 104).

En este orden de ideas, se planteó un estudio con el objeto de fortalecer la competencia resolución de problemas en estudiantes de 70 grado de un colegio público rural, ubicado en el departamento de Santander-Colombia, mediante implementación de una secuencia didáctica basada en el método de Pólya y la aplicación de la metodología del Aprendizaje Basado en Problemas (ABP), usando como contexto la enseñanza de los números enteros.

\subsection{Aspectos teóricos}

En este apartado se abordan de manera sucinta los lineamientos teóricos que guiaron la investigación: la secuencia didáctica, el APB, la competencia resolución de problemas, el método de Pólya y los números enteros.

La secuencia didáctica para Díaz-Barriga (2013) es de preponderante importancia en el proceso de planeación y gestión de la clase, es por ello que propone un modelo de secuencia didáctica que se fundamenta en tres momentos: actividades de apertura, actividades de desarrollo y actividades de finalización. Los tres momentos deben estar relacionados entre sí, vinculados a situaciones problemáticas y contextos reales, y corresponden a las necesidades de aprendizaje identificadas antes por el docente.

Por su parte, García, López \& Peña (2014) describen el ABP como una metodología de gran importancia en el proceso enseñanza-aprendizaje y una estrategia centrada en la búsqueda del interés y la promoción de aprendizajes en los estudiantes. Además, identifican tres momentos: antes, durante y después de la clase, y cinco fases: escenario o problema, comprensión del problema, búsqueda de alternativas, selección de la mejor 
alternativa y escenario de ejecución del ABP. Los mismos autores plantean, entre otras características, que la estrategia ABP promueve la responsabilidad del aprendizaje por parte del estudiante, fortalece el trabajo en grupo y genera habilidades para la búsqueda de información.

Entre tanto, Santos (2014) describe la importancia de la resolución de problemas en las diferentes etapas del aprendizaje, indicando que enseñar la competencia resolución de problemas debe ser el centro de la enseñanza de las matemáticas; además señala que para resolver situaciones problemáticas se debe enseñar métodos generales y particulares, el uso de heurísticas, la conexión con otras áreas del conocimiento y la creación de una comunidad matemática en el salón de clases.

Los trabajos de Pólya (1945/1985, 1962/1981, 1954a, 1964b, 1962/1981, 1989), ampliamente conocidos, se enfocan principalmente en el desarrollo de estrategias para la resolución de problemas, resaltan un método que consiste en una secuencia de cuatro pasos lógicos que se pueden aplicar a cualquier problema: comprender el problema, concebir un plan, ejecutar el plan y examinar la solución obtenida. Además, el método es de fácil enseñanza por su gran aplicabilidad y cada paso cumple una función especifica para resolver el problema, y al mismo tiempo están estrechamente relacionados entre sí.

Los aspectos teóricos mencionados hasta el momento conforman una posible amalgama de saberes que permiten el desarrollo de actitudes, disposiciones y habilidades para el abordaje de problemas matemáticos hasta lograr su solución.

En relación al contexto para el desarrollo de la competencia resolución de problemas, se tuvo en cuenta el concepto de número entero desde las líneas de Vargas, et al. (1999), que proporcionan un imaginario de número entero más amplio que el que se asume en las prácticas tradicionales de aula. Es decir, asumirlos como parte de la cultura matemática ligados al entorno social y no únicamente como una herramienta matemática necesaria para desarrollar contenidos posteriores; así como también, permitir que los estudiantes conozcan y descubran las aplicaciones y utilidades de los números enteros en contextos más de la cotidianidad que en contextos netamente matemáticos.

A continuación, se presenta la figura 1 en la que se relacionan los principales constructos teóricos que permitieron el desarrollo de esta investigación: Díaz-Barriga (2013), García, López \& Peña (2014), Santos (2014), Pólya (1989) y Vargas et al. (1999).

Figura 1

Relación entre los constructos teóricos usados en la investigación

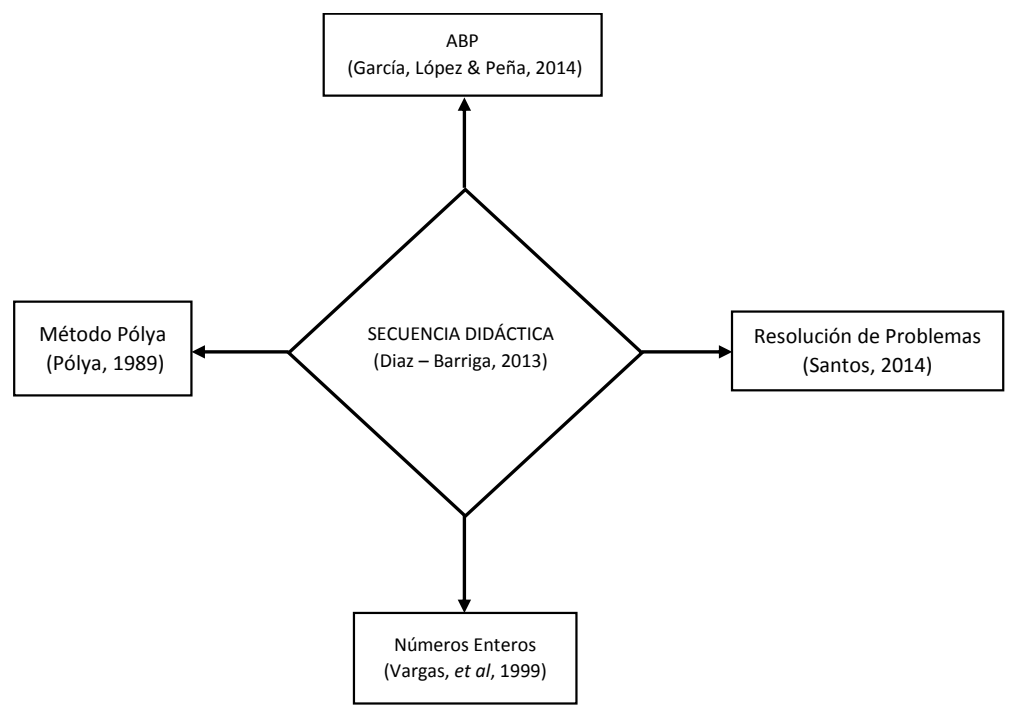

Fuente: elaboración propia 
En la figura 1 se observa que los diferentes constructos están relacionados entre sí, esto se debe a que durante toda la investigación están presentes de manera directa o indirecta; pero todas inmersas en la secuencia didáctica, la cual determina las diferentes fases de la investigación y la intensidad con la cual se desarrolla cada uno de los anteriores teóricos.

En concreto, todas estas ideas presentadas por los diferentes teóricos están en armonía con los objetivos y el desarrollo del estudio, los cuales permiten fortalecer la competencia resolución de problemas.

\section{Metodología}

\subsection{Tipo de estudio}

La metodología de investigación es de tipo Investigación-Acción con enfoque cualitativo, por cuanto facilita el desarrollo de las diferentes actividades de forma estructurada y secuencial, al tiempo que se fortalece el pensamiento matemático, pensamiento crítico y la reflexión. El grupo participante estaba conformado por 30 estudiantes de grado $7^{\circ}$ del colegio elegido para el estudio.

\subsection{Instrumentos de recolección de información}

\section{Pruebas diagnósticas}

Se realizaron dos pruebas diagnósticas, una al iniciar y la otra al finalizar la secuencia didáctica. La primera prueba permitió la identificación de dificultades de los estudiantes y permitió establecer los objetivos de aprendizaje de acuerdo a las necesidades de aprendizaje de los estudiantes. La segunda prueba permitió medir el impacto de la aplicación de la secuencia didáctica en el aprendizaje de los estudiantes al solucionar situaciones problemáticas.

\section{Actividades: enseñanza del método de Pólya}

Se aplicaron cuatro actividades que tenían como objetivo la enseñanza y aplicación del método de Pólya. Las actividades estaban constituidas por una serie de situaciones problemáticas, en diferente presentación y contextos. Cada actividad se aplicó durante una sesión de 110 minutos. Además, se enseñaron algunas heurísticas que permitieron el desarrollo de habilidades para solucionar situaciones problemáticas.

\section{Actividades: enseñanza del conjunto de los números enteros con la metodología del ABP}

Se aplicaron cuatro actividades que tenían como objetivo la enseñanza del concepto y orden de los números enteros, y las operaciones de adición, sustracción y multiplicación. Las actividades estaban dividas en tres partes: la primera era una serie de problemas relacionadas con el objeto de estudio que se desarrolla en la guía, la segunda era la presentación del objeto de estudio y, la tercera, otras situaciones problemáticas que permitían afianzar y aplicar los aprendizajes que se enseñaron en la guía. La metodología del ABP se aplicó con grupos de a dos o tres estudiantes.

\section{Diario de campo}

El diario de campo permitió describir el desarrollo de las clases y los sentires de los estudiantes durante la implementación de las actividades y las pruebas diagnósticas. A partir de las descripciones realizadas se logró mejorar, enriquecer y transformar las prácticas de clase mientras se desarrollaba la secuencia didáctica (Meléndez, 2017).

\section{Resultados}

\subsection{Procedimiento de análisis de datos}

Se analizaron las respuestas de los estudiantes de acuerdo a las estrategias que empleaban y nivel de acierto que tenían en la solución de preguntas o situaciones problemáticas. Así mismo se utilizó una tabla de categorías que 
permitieron identificar el desempeño de los estudiantes al solucionar situaciones problemáticas, el trabajo en equipo, la pertinencia del material pedagógico y desempeño del docente durante la aplicación de la secuencia didáctica. A continuación la tabla 1 muestra las categorías, subcategorías e indicadores que permiten la asignación de las mismas las producciones de los estudiantes.

Tabla 1

Categorías, subcategorías e indicadores de análisis

\begin{tabular}{|c|c|c|}
\hline Categorías & Subcategorías & Indicadores \\
\hline \multirow{3}{*}{ Resolución de problemas } & Interpretación & $\begin{array}{l}\text { Comprende todos los elementos del problema antes de comenzar a } \\
\text { resolverlo. }\end{array}$ \\
\hline & Algoritmos & Identifica la operación que debe realizar para encontrar la solución. \\
\hline & Desempeño & Realiza correctamente la operación. \\
\hline \multirow{4}{*}{$\begin{array}{l}\text { Resolución de problemas } \\
\text { método de Pólya }\end{array}$} & $\begin{array}{l}\text { Comprensión } \\
\text { del problema }\end{array}$ & $\begin{array}{l}\text { Determina la incógnita, los datos, las condiciones, y decidir si esas } \\
\text { condiciones son suficientes, no redundantes ni contradictorias. }\end{array}$ \\
\hline & $\begin{array}{l}\text { Concebir un } \\
\text { plan }\end{array}$ & $\begin{array}{l}\text { Plantea una estrategia o utiliza diferentes métodos heurísticos para } \\
\text { solucionar el problema. }\end{array}$ \\
\hline & $\begin{array}{l}\text { Ejecución del } \\
\text { plan }\end{array}$ & $\begin{array}{l}\text { Implementa el plan concebido en el paso anterior y examina cada paso } \\
\text { que conllevan a la solución del problema este correcto. }\end{array}$ \\
\hline & $\begin{array}{l}\text { Examinar la } \\
\text { solución }\end{array}$ & Observa que fue lo que se hizo; verificando el resultado y razonamiento. \\
\hline \multirow[b]{2}{*}{ Trabajo en equipo } & Disponibilidad & Manifiesta agrado al realizar actividades en equipo de trabajo. \\
\hline & Asertividad & Se desenvuelve eficientemente realizando actividades en equipo. \\
\hline \multirow{2}{*}{ Práctica pedagógica } & $\begin{array}{l}\text { Diseño de } \\
\text { recursos } \\
\text { (actividades) }\end{array}$ & $\begin{array}{l}\text { Las guías de aprendizaje se relacionan con el problema y los objetivos de } \\
\text { la investigación. }\end{array}$ \\
\hline & $\begin{array}{l}\text { Estrategias } \\
\text { pedagógicas }\end{array}$ & $\begin{array}{l}\text { Los procesos que el estudiante debe realizar se explican de forma clara y } \\
\text { coherente. }\end{array}$ \\
\hline
\end{tabular}

Fuente: adaptación de Meneses y Peñaloza (2017)

\subsection{Análisis de la prueba diagnóstica inicial}

La prueba diagnóstica consistió de 10 situaciones problemáticas de diferente complejidad, en la cual los estudiantes disponían de un tiempo de 110 minutos para su desarrollo. En la prueba se utilizaron problemas no rutinarios para que los estudiantes pudieran emplear diferentes recursos o estrategias para su solución. Dentro de los resultados se puede destacar:

i) Las situaciones no fueron resueltas en su totalidad, según lo manifestado por los estudiantes, porque no estaban acostumbrados a resolver este tipo de problemas.

ii) Las respuestas fueron cortas y con justificaciones muy deficientes.

iii) Se encontró que los estudiantes tienen diversas dificultades: aplicar mal un algoritmo, una deficiente interpretación del problema y ejecución de estrategias de solución, uso equívoco de imágenes que justifican la solución de un problema. Este tipo de respuestas las podemos observar en las figuras 2 y 3 . Muchas de estas dificultades se deben a que los docentes le han dado prioridad a enseñar un algoritmo y aplicarlo en ejercicios similares, lo que les cohibe de mejorar sus razonamientos. 
Figura 2

Algunas respuestas del problema 1 de la prueba diagnóstica inicial

1. Si subo una escalera de dos en dos peldaños, doy nueve pasos más que subiendo de tres en tres ¿Cuántos peldaños tiene la escalera?

tengo que escakar 3 Peldaños

1. Si subo una escalera de dos en dos peldaños, doy nueve pasos más que subiendo de tres en tres ¿Cuántos peldaños tiene la escalera?

Tiene nueve escalones

1. Si subo una escalera de dos en dos peldaños, doy nueve pasos más que subiendo de tres en tres ¿Cuántos peldaños tiene la escalera?

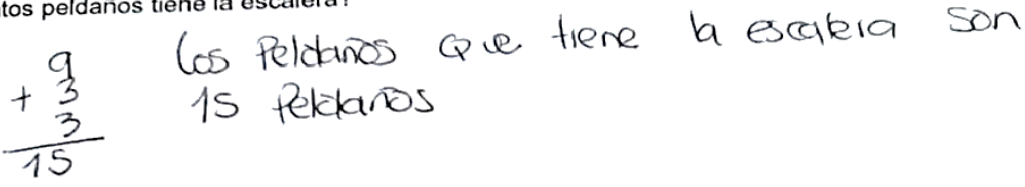

1. Si subo una escalera de dos en dos peldaños, doy nueve pasos más que subiendo de tres en tres ¿Cuántos peldaños tiene la escalera? 12 peldaños

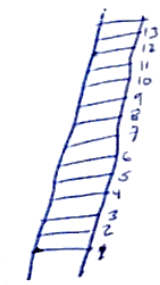

Fuente: ejercicios desarrollados por los estudiantes participantes en la investigación

Figura 3

Algunas respuestas de los problemas 7 y 10

de la prueba diagnóstica inicial

7. Observa la secuencia y elige la Necha que slgue. Justifica tu respuesta.

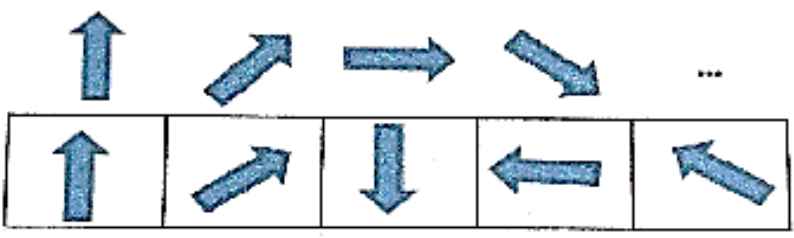
A
B
(C)
D
E

10 Fo una fiesta infantil se ha reunido regalos para un cierto número de niños de forma quo a cada nría le corresponde tres regalos. A la hora de la fiesta se presenta la quinta parte de los nifhos privistos Suponiendo que so reparten todos los premios entre los nif́os asistentes ¿cuántos conresponderát a cada nifo?

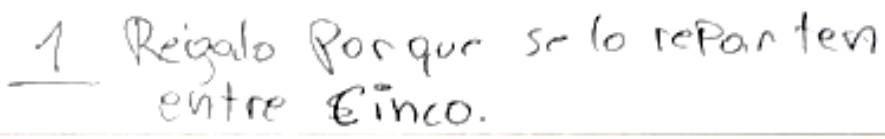

10. En una fiesta infantil se ha reunido regalos para un cierto número de niños de forma que a cada niño le corresponde tres regalos. A la hora de la fiesta se presenta la quinta parte de los nif̂os previstos. Suponiendo que se reparten todos los premios entre los niños asistentes ¿cuántos corresponderá a cada nif̂o?

de ha $S$ recidos para cada nitio

Fuente: ejercicios desarrollados por los estudiantes participantes en la investigación 


\subsection{Análisis de las actividades de enseñanza del método de Pólya}

Teniendo en cuenta las dificultades que puedan presentar los estudiantes, se incorpora a la estrategia el método Pólya, el cual comprende una estructura clara y sistemática para resolver problemas matemáticos: entender el problema, configurar un plan, ejecutar el plan y analizar la solución obtenida; en cada una de ellas el docente puede plantear una serie de cuestionamientos que pueden guiar al estudiante, alcanzando con ello una aprendizaje significativo mediante la reflexión y el pensamiento crítico.

Se elaboraron cuatro actividades correspondientes a la enseñanza del método de Pólya y cada actividad se aplicó durante una sesión de clase. En algunas de las actividades se enseñaron diferentes heurísticas con el objetivo de brindar a los estudiantes diferentes estrategias cuando fueran a solucionar una situación problemática. Inicialmente los estudiantes presentaron dificultades en describir en cada uno de los pasos del método de Pólya, el cómo solucionaban los problemas; esta dificultad la podemos evidenciar en la figura 4, donde los estudiantes solucionan el problema, aunque no de forma correcta y no describen en las casillas los procedimientos o elementos que utilizaron.

\section{Figura 4}

Algunas respuestas de las actividades enseñanza del metodo de Pólya

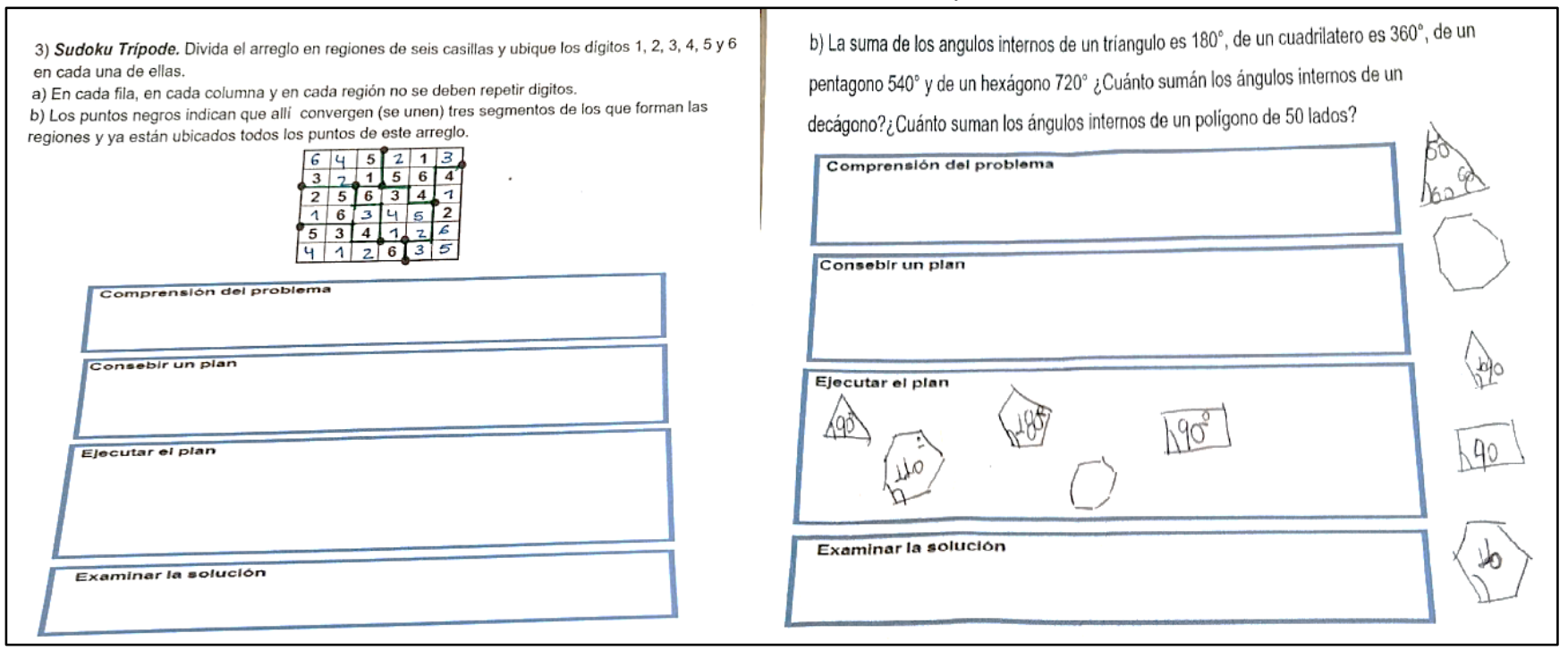

Fuente: ejercicios desarrollados por los estudiantes participantes en la investigación

El docente explicó la importancia de describir en las casillas lo que hacía cada estudiante para solucionar el problema. Esto permitió que los estudiantes aprendieran mejor el método estudiado y mejorar los resultados de los estudiantes al solucionar las situaciones problemáticas, como se evidencia en la figura 5. Algunos de los estudiantes desarrollaron estas actividades en grupos de máximo tres estudiantes y otros de manera individual; en la figura 5 podemos observar que un estudiante también agregó las respuestas de sus dos compañeros. 
Figura 5

Algunas respueestas de las actividades enseñanza del método de Pólya

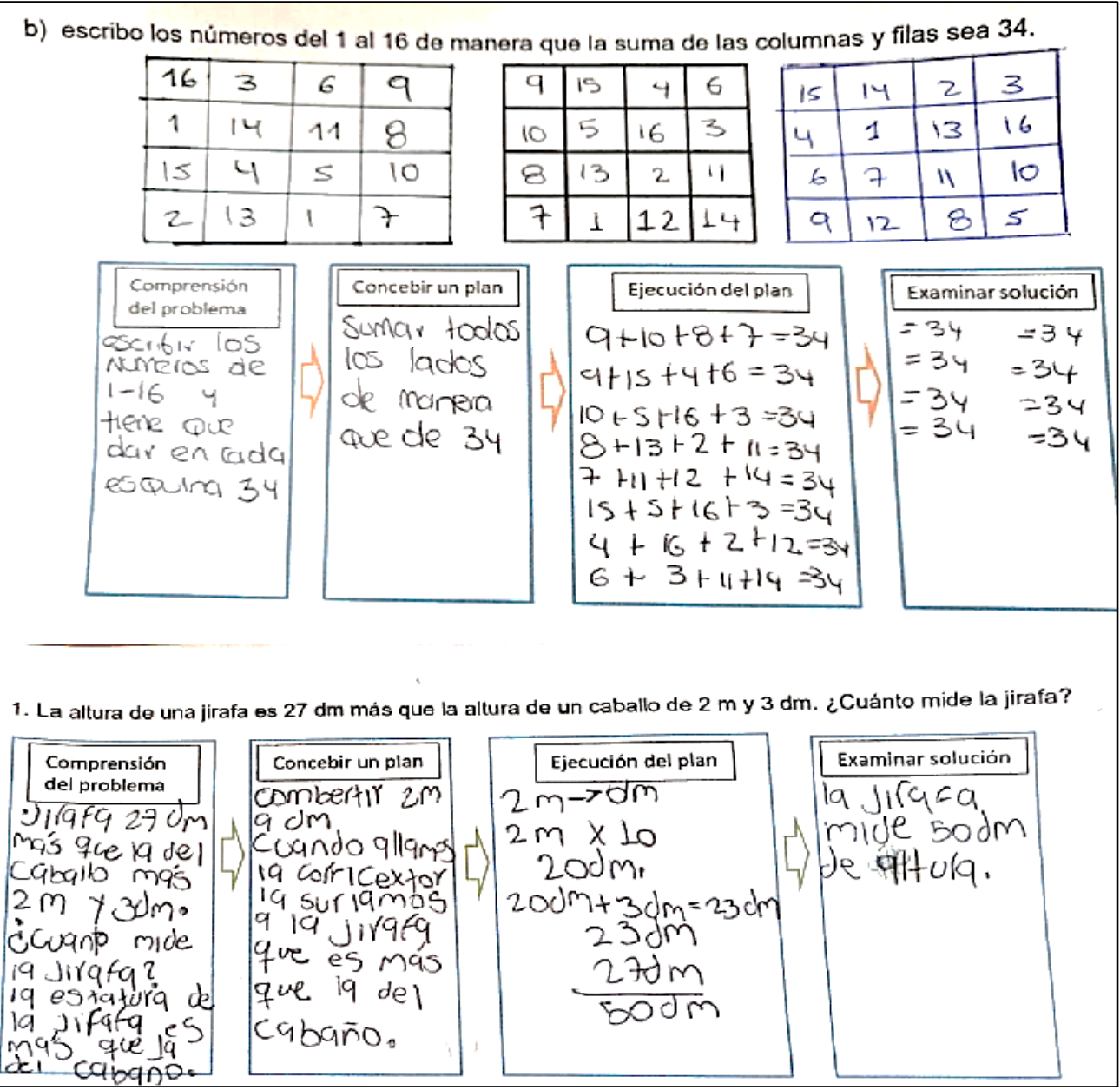

Fuente: ejercicios desarrollados por los estudiantes participantes en la investigación

\subsection{Análisis de las actividades enseñanza del conjunto de los números enteros y metodología del ABP}

Se elige el ABP, como estrategia didáctica, por cuanto permite contextualizar diversas situaciones problemáticas, facilitando la adquisición y generación de conocimientos y competencias en los estudiantes, en especial aquellas de gran complejidad como la resolución de problemas y los procesos articulados a esta (modelado, comunicación, razonamiento, formulación, comparación y ejercitación de procedimientos) (MEN, 2017), pues se centra en el aprendizaje más que en la solución, acercando al estudiante al tipo de problema que debe afrontar posiblemente en un futuro (Subdirección de Currículum y Evaluación, Universidad Tecnológica de Chile INACAP, 2017).

Las actividades que se aplicaron en esta investigación con el enfoque del ABP estaban constituidas por cuatro partes; la primera con un conjunto de situaciones problemáticas relacionadas con la temática a estudiar en la actividad; la segunda con el concepto y explicación de algunos ejercicios y situaciones problemáticas resueltas del objeto matemático de estudio y, para finalizar, más ejercicios y situaciones problemáticas.

Los estudiantes presentaron dificultades en las primeras actividades, principalmente porque para darles solución la información se encontraba al inicio de la misma y los estudiantes no se percataban de ello. Esta dificultad 
persistió en algunos estudiantes durante algunas sesiones, como lo muestra las figuras 6 y 7 , donde el estudiante no logra diferenciar completamente los números enteros positivos y negativos, y sus aplicaciones.

Figura 6

Algunas respuestas de las actividades de la metodología del ABP

1. Escribir los números enteros que corresponden a cada letra

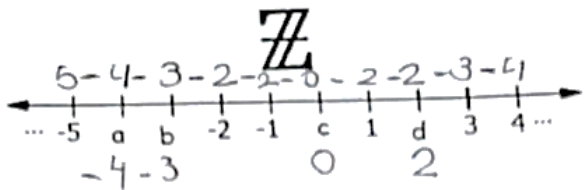

2. Asigno un número entero a cada situación

- En una ciudad el termómetro marcaba $-5^{\circ} \mathrm{C}$ y sois más tarde marco $8^{\circ}$ ¿Cuánto fue el cambio de temperatura? $\perp 4^{\circ} \mathrm{C}$

- Si se invirtieron 30000 euros en un negocio y se recogieron 18000 . ¿Cuánto fue la ganancia? 18.000

- Un número mayor que -7 y menor que $5 .=0$

- El año 250 antes de Cristo. $=249$

3. De acuerdo con el cálculo aproximado de cada temperatura, ordénalos de menor a mayor en la segunda columna.

a) Temperatura del polo sur.

b) Temperatura promedio del lugar donde vives.

c) Temperatura de una estrella.

d) Punto de congelación del agua.

e) Punto de ebullición del agua.

f) Temperatura promedio del desierto de La Tatacoa.

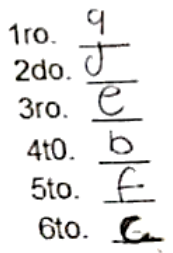

4. halla el valor de la letra
a) $|a|=5$
b) $|-1|=x \downarrow$
c) $|d+1|=6$
d) $|m-4|=0$
$5 \quad-4$
e) $\begin{gathered}|4-n|=1 \\ 7\end{gathered}$

Fuente: ejercicios desarrollados por los estudiantes participantes en la investigación

Figura 7

Algunas respuestas de las actividades de la metodología del ABP

d) A continuación se da una lista de las personas que más influyeron en las matemáticas.

\begin{tabular}{|l|c|c|}
\hline \multicolumn{1}{|c|}{ Nombre } & Nació hace: & Fecha de nacimiento \\
\hline Pitágoras de Samos & 2589 años & 570 \\
\hline Euclides & 2384 años & 0365 \\
\hline Carl Gauss & 242 años & 2223 \\
\hline Arquimides & 2306 años & 2287 \\
\hline Leonhard Euler & 236 años & 377 \\
\hline Isaac Newton & 377 años & 2358 \\
\hline
\end{tabular}

Completa la tabla sabiendo que el año 0 es hace 2019 años

\begin{tabular}{|c|c|c|c|c|c|}
\hline $\begin{array}{l}2589 \\
2019\end{array}$ & $\begin{array}{l}2384 \\
2019\end{array}$ & $\begin{array}{r}242 \\
2019\end{array}$ & $\begin{array}{l}2306 \\
2019\end{array}$ & $\begin{array}{r}236 \\
2019\end{array}$ & $\begin{array}{r}377 \\
2019\end{array}$ \\
\hline 0570 & 0365 & & & 2217 & \\
\hline
\end{tabular}

Fuente: ejercicios desarrollados por los estudiantes participantes en la investigación 
En la figura 8, se observa que los estudiantes, tras algunas sesiones de clase, logran alcanzar respuestas más elaboradas y de acuerdo a lo que se solicita en la pregunta.

Figura 8

Algunas respuestas de las actividades de la metodología del ABP

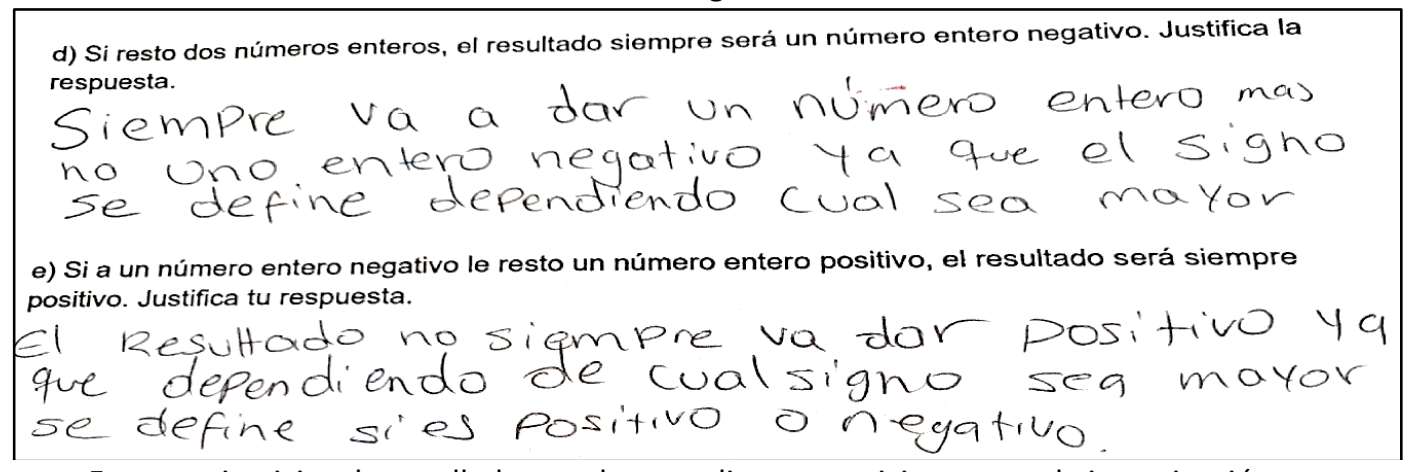

Fuente: ejercicios desarrollados por los estudiantes participantes en la investigación

Al finalizar la aplicación de estas cuatro actividades, y luego de analizar su ejecución, se puede concluir que los estudiantes mejoraron en sus desempeños; por ejemplo, gracias al ABP, el trabajo en grupo e individual se fortaleció, al igual que las habilidades metacognitivas y autonomía de los estudiantes, lo que da cuenta de los beneficios de esta metodología (García, López \& Peña, 2014). En este sentido, se desarrollaron y fortalecieron el pensamiento crítico y reflexivo, el trabajo cooperativo y en equipo, la autonomía y la autogestión del aprendizaje. Por otro lado, con el método de Pólya y las diferentes heurísticas se logró que los estudiantes adquieran una serie de herramientas que les son útiles en la solución de situaciones problemáticas.

Los beneficios de aprender a resolver problemas son diversos e importantes, por medio de las actividades desarrolladas los estudiantes lograron comprender que los problemas no están separados de los algoritmos y conceptos que se aprenden sobre una temática y, mucho menos, de las situaciones que viven ellos y la sociedad, lo cual permite confirmar lo expuesto por Santos (2014), los estudiantes entienden los propósitos y usos del conocimiento adquirido, así mismo deducen que lo pueden hacer en cualquier contexto.

\subsection{Análisis de la prueba diagnóstica final}

Los resultados de la prueba diagnóstica final dan cuenta de lo aprendido por los estudiantes, puesto que las actividades propuestas fueron construidas con base a todo lo estudiado durante la secuencia didáctica. No obstante, el interés del estudiante por dar respuesta a cada uno de los ejercicios, existieron varios factores que interfirieron, entre ellos: la falta de tiempo para contestar la prueba (que es individual y no en grupo), el miedo que tienen algunos estudiantes siempre que presentan una prueba, y que no utilicen información de apoyo, como las guías para desarrollar la prueba.

De acuerdo con lo anterior, el Gobierno de Navarra (2008) indica que:

La evaluación diagnóstica no es una prueba que evalúa todo lo que ha aprendido el alumno: debido a su carácter puntual y limitado, deja fuera de la evaluación aprendizajes muy útiles y valiosos realizados. De ahí la complementariedad de esta evaluación con la continua que realiza el profesorado (p.16).

La recolección de información sobre lo realizado por los estudiantes en las diferentes actividades permitió aplicar la evaluación continua y sumativa, que permite analizar el proceso de aprendizaje individual y colectivo del grupo de estudio. 
Al comparar la prueba diagnóstica final con la inicial, se encuentran cambios significativos en las respuestas de los estudiantes: respuestas más elaboradas y estrategias claras para solucionar una situación problemática. Así como también, se observó que el porcentaje de preguntas sin respuesta es menor en la prueba final que en inicial; de manera similar, disminuyó el número de consultas que los estudiantes hacían al docente durante la implementación de las actividades. A continuación, las figuras 9 y 10 muestran las producciones de estudiantes en donde se observa el avance positivo de los estudiantes al resolver problemas en la prueba diagnóstica final.

Figura 9

Algunas respuestas de la prueba diagnóstica final

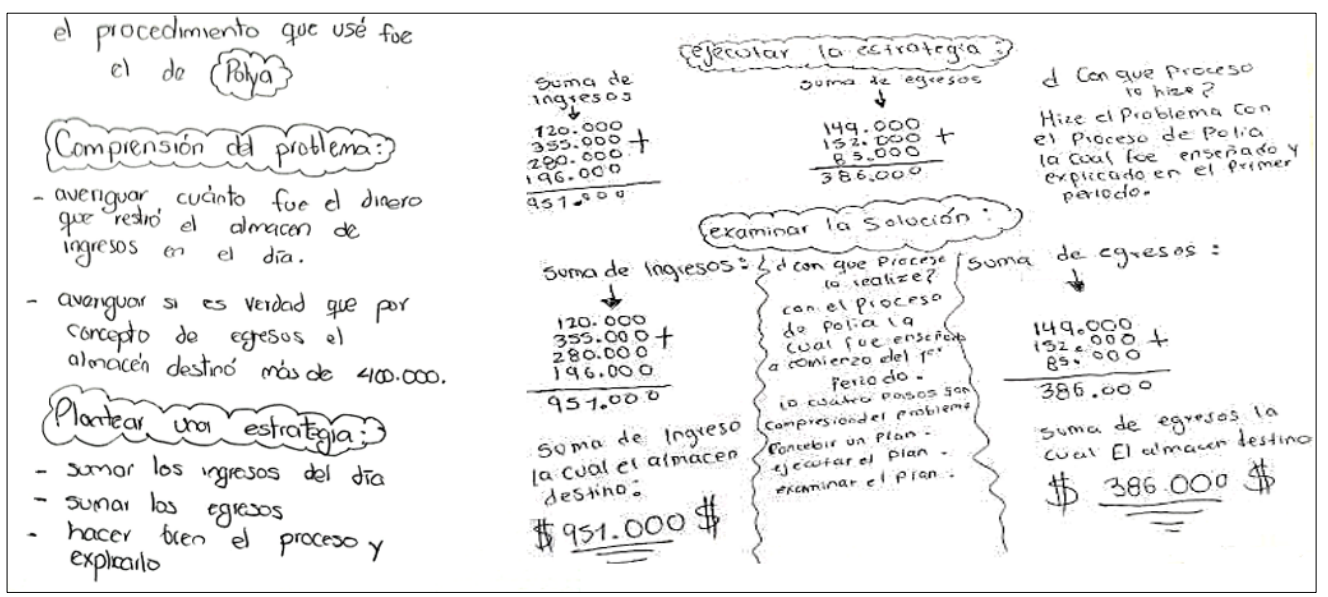

Fuente: ejercicios desarrollados por los estudiantes participantes en la investigación

Figura 10

Algunas respuestas de la prueba diagnóstica inicial 2. Divide la figura en dos partes congruentes. Las partes no deben tener forma rectangular, luego
ubica números enteros positivos y negativos diferentes, de tal manera que cada parte la suma de sea igual
$\begin{array}{r}1 p \\ 9 \\ \frac{3}{6} \\ \hline 18\end{array}$
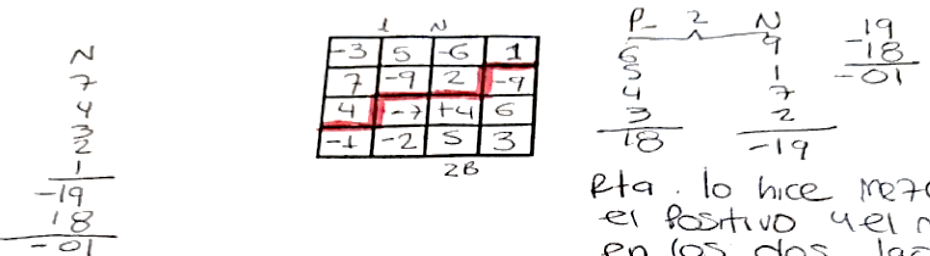
Rta. lo hice mezclando el Positivo yel negativo en los dos lados.

3. Separa los números en tres grupos. Sin modificar su orden. Hazlo de tal manera que cuando sumes los números de cada grupo el resultado sea el mismo.
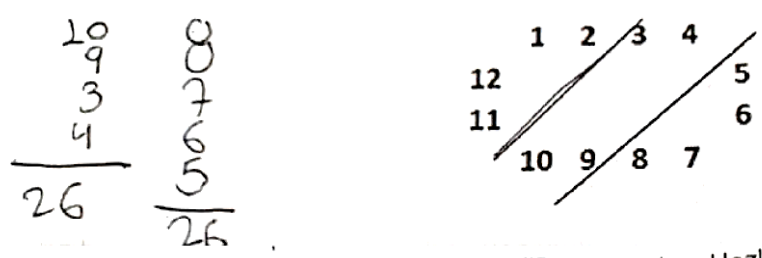

Rita=hice partlendo IaS tres lajos sumando les numelos,

en tres grupos. Sin modificar su orden. Hazlo de tal manera que cuando sumes los números de cada grupo el resultado sea el mismo.

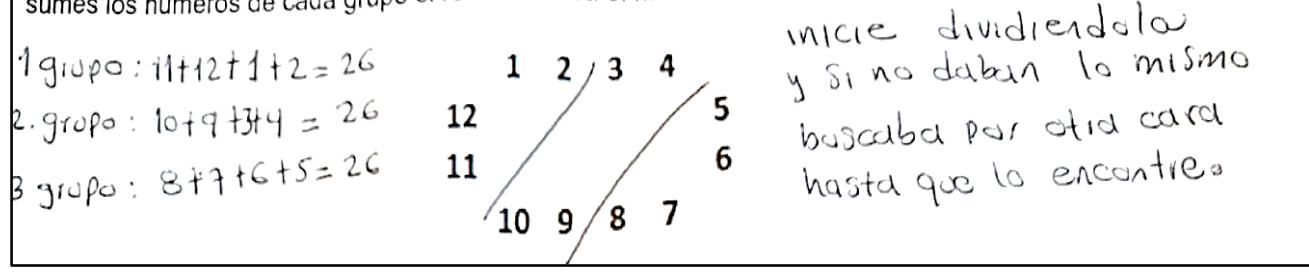

Fuente: ejercicios desarrollados por los estudiantes participantes en la investigación 
Todos estos factores demuestran mayor seguridad al contestar, mejor aplicación de lo aprendido y mejor interpretación de cada situación por parte de los estudiantes. Además, como las actividades fueron desarrolladas en pequeños grupos (dos o tres estudiantes), facilitó su participación al momento de expresar opiniones o realizar aportes en las diferentes discusiones que surgieron. Igualmente se observó mayor responsabilidad respecto al rol que cumplían dentro del grupo, pues al ser pocos sus integrantes tendrían más oportunidad de participar; estas características las resalta Schoenfeld (1985, citado por Santos, 2014), quien considera que se tienen experiencias más significativas al fomentar discusiones en pequeños grupos para la solución de problemas, en donde el docente actúa como guía y orientador de estas.

\section{Conclusiones}

En este capítulo se presentan las principales conclusiones del estudio abordado, fruto de los análisis y resultados realizados.

\subsection{En relación a la prueba diagnóstica inicial}

Los análisis y resultados de la prueba diagnóstica inicial muestran que los estudiantes tenían dificultades en comprender un problema, utilizar estrategias para su solución y los estudiantes tenían algunas falencias en los presaberes. Todo esto conllevó a que las respuestas de los estudiantes fueran cortas, algunas sin justificación, no utilizaban todos las condiciones o datos presentes en la situación problemática y a un bajo acierto de las respuestas con respecto al problema a solucionar. Además, se logró identificar que los estudiantes no utilizaban un método o un procedimiento definido para solucionar problemas.

\subsection{Enseñanza del método de Pólya}

Enseñar el método de Pólya, el cual es una estrategia clara, con pasos lógicos, secuenciales y sencillos para solucionar problemas, permitió a los estudiantes un mayor éxito en la solución de las situaciones matemáticas planteadas. Además, algunos estudiantes relacionaban el nuevo método aprendido con las estrategias que utilizaban para solucionar problemas. Todo esto permitió una gran aceptación por parte de los estudiantes hacía el método de Pólya, debido a que muchas de las dificultades que tenían, y habían sido identificadas en la prueba diagnóstica inicial, fueron superadas por los estudiantes (entre ellas se puede destacar la justificación apropiada de las respuestas a las preguntas del problema, asociación de condiciones iniciales con los datos, con las respuestas y las estrategias usadas para solucionar lo planteado).

\subsection{Aplicación de la metodología del ABP}

Los estudiantes lograron aprender las diferentes temáticas desarrolladas sobre los números enteros, demostrando que la metodología del ABP se puede enseñar con cualquier objeto de estudio y cualquier grado académico. También permitió fortalecer el trabajo autónomo y en grupo, fortaleciendo el liderazgo, la toma decisiones, organización de actividades e ideas de los estudiantes y consecuentemente el aprendizaje de la temática de estudio. Además, la metodología permitió que los estudiantes interactuaran en todo momento con situaciones problemáticas, logrando que los estudiantes relacionaran concepto, algoritmo y aplicaciones como la principal fuente de aprendizaje y no como tres elementos independientes entre sí y con poca relación.

Es importante resaltar que una de las dificultades que se presentó se debió a que la guía, al estar estructurada en tres partes (situaciones problemáticas, objeto matemático a estudiar y ejercicios de práctica con problemas), condujo a algunos estudiantes a solucionar la primera parte sin tener los conocimientos necesarios del objeto matemático y así acarrear errores principalmente aritméticos al solucionar los problemas; demostrando que a los estudiantes todavía se les dificulta seguir las instrucciones dadas por el docente. 


\subsection{En relación a la socialización de respuestas durante la clase y el diario de campo}

La socialización de respuestas, por parte de los estudiantes durante las clases en las diferentes actividades, fue la principal herramienta de reflexión que permitió identificar dificultades y avances de los estudiantes. Además, ayudó a motivar a los estudiantes a compartir sus saberes, generando interés y motivación a solucionar problemas. También, el diario de campo permitió describir el desarrollo de las diferentes actividades en el salón de clase; evidenciando que es una herramienta útil al docente, para la identificación de falencias, ritmos de aprendizaje y fortalezas de los estudiantes. Es importante que el docente utilice el diario de campo para el registro de los sucesos de la clase y así reflexionar sobre práctica y gestionar la mejoras necesarias. En concreto, aprovechar las bondades de esta herramienta de recolección de información para avanzar en los procesos de mejora constante.

\subsection{Prueba diagnóstica final}

Los análisis de los resultados de la prueba diagnóstica final permitieron demostrar que los objetivos planteados en esta investigación fueron alcanzados, ya que se fortaleció la competencia resolución de problemas. Esto se evidencia en que las respuestas de los estudiantes, al resolver los problemas de esta prueba fueron más elaboradas que la prueba diagnóstica inicial. La utilización correcta de diversas estrategias en solucionar problemas conllevó a un gran éxito en las respuestas. Los conceptos evaluados, que fueron enseñados por la metodología del ABP y el método Pólya, tuvieron una exitosa apropiación por parte de los estudiantes. Es por ello que es posible sugerir que los resultados han sido muy positivos y se espera a futuro que se vean reflejados en las pruebas tanto internas como externas de la institución educativa.

\section{Referencias bibliográficas}

Alvarez, M. (2020). Aprendizaje situado: mito o realidad desde el aprendizaje basado en problemas. Revista Espacios, 41(28), 26. Recuperado de: https://www.revistaespacios.com/a20v41n28/a20v41n28p26.pdf

Callejo, L. (1998). Un club matemático para la diversidad. Madrid, España: Narcea.

Cockcroft, W. H. (1982). Mathematics counts. London, United Kingdom: Her Majesty's Stationery Office.

Díaz-Barriga, A. (2013). Secuencias de aprendizaje. ¿Un problema del enfoque de competencias o un reencuentro con perspectivas didácticas? Revista de Currículum y Formación de Profesorado, 17(3), 11-33. Recuperado de: https://www.redalyc.org/pdf/567/56729527002.pdf

García, J., López, N., \& Peña, A. (2014). El aprendizaje basado en problemas (ABP) Conectar aprendizajes con la vida. Ciudad de Máxico, México: Editorial Instituto de Estudios Superiores en Educación por Competencias S.C.

Gobierno de Navarra, Departamento de Educación. (2008). La evaluación diagnóstica: características. Marco teórico de la evaluación diagnóstica. Educación primaria. Recuperado de: https://docplayer.es/15849942Sevaluacion-marco-teorico-de-la-evaluacion-diagnostica-del-sistema-educacion-primaria.html

Meléndez, S. (2017). Implementación de una estrategia pedagógica basada en el método Pólya, para el mejoramiento de la competencia interpretativa en la resolución de problemas matemáticos, en estudiantes de quinto grado del Colegio Gustavo Cote Uribe. (Tesis de maestría). Universidad Autónoma de Bucaramanga, Bucaramanga, Colombia.

Meneses, M., \& Peñaloza, D. (2017). Método de Polya como estrategia pedagógica para fortalecer la competencia resolución de problemas matemáticos con operaciones básicas en estudiantes de los grados 
tercero y cuarto del colegio municipal aeropuerto. (Tesis de Maestría). Universidad Autónoma de Bucaramanga, Bucaramanga, Colombia.

Ministerio de Educación Nacional de Colombia, MEN. (1998). Lineamientos Curriculares Matemáticas. Santa Fe de Bogotá, Colombia: Delfín.

Ministerio de Educación Nacional de Colombia, MEN. (2017). Mallas de Aprendizaje. Documento para la implementación de los DBA. Bogotá. Recuperado de: https://aprende.colombiaaprende.edu.co/ckfinder/userfiles/files/CARTILLA-INTRODUCTORIA_.pdf

Organisation for Economic Co-operation and Development, OECD (2006). Assessing Scientific, Reading and Mathematical Literacy: A Frameworkfor PISA 2006. París. OECD, http://www.pisa.oecd.org/. Traducción castellana(2006), Evaluación de la Competencia Científica, Lectora y Matemática: Unmarco teórico para PISA 2006. Madrid: INECSE/MEC,http://www.ince.mec.es/index.htm.

Pólya, G. (1945/1985). How to solve it: A new aspect of mathematical method (2nd ed.). Princeton, NJ: Princeton University Press.

Pólya, G. (1954a). Mathematics and plausible reasoning: Vol. 1. Induction and analogy in mathematics. Princeton, NJ: Princeton University Press.

Pólya, G. (1954b). Mathematics and plausible reasoning: Vol. 2. Patterns of plausible inference. Princeton, NJ: Princeton University Press.

Pólya, G. (1962/1981). Mathematical discovery: On understanding, learning, and teaching problem solving. New York: John Wiley \& Sons.

Pólya, G. (1989). Cómo plantear y resolver problemas. Ciudad de México, México: Trillas.

Restrepo, B. (2005). Aprendizaje basado en problemas (ABP): una innovación didáctica para la enseñanza universitaria. Revista Educación y Educadores 8, 9-19.

Santos, L. (2014). La resolución de problemas matemáticos fundamentos cognitivos. Ciudad de México, México:

Trillas Editorial.

Schoenfeld, A. (1985). Mathematical Problem Solving. New York, EE.UU: Academic Press.

Subdirección de Currículum y Evaluación, Universidad Tecnológica de Chile INACAP. (2017). Manual de Estrategias Didácticas: Orientaciones para su selección. Santiago de Chile: Ediciones INACAP. Recuperado de: http://www.inacap.cl/web/2018/documentos/Manual-de-Estrategias.pdf

Valdez-Rojo, E, \& Tobón, S. (2018). Diseño de situaciones de aprendizaje para la resolución de problemas con base en las matemáticas desde la socioformación. Revista Espacios. 39(53), 18. Recuperado de: http://www.revistaespacios.com/cited2017/cited2017-18.pdf

Vargas-Machuca, I., Jimeno, M., Iriarte, M., González, J., Ortiz, A., Sanz, E. \& Ortiz, A., (1999). Números enteros. Madrid, España: Editorial Síntesis.

Esta obra está bajo una Licencia Creative Commons Attribución-NoCommercial 4.0 International

(cc) BY-NC 